\title{
A THEOREM IN OPERATIONAL CALCULUS AND SOME INTEGRALS INVOLVING LEGENDRE, BESSEL AND $E$-FUNCTIONS
}

\author{
by C. B. RATHIE
}

(Received, 11th April, 1955)

1. In this paper we prove a theorem in Operational Calculus and use it to evaluate a few infinite integrals involving Legendre, Bessel and $E$-functions. We write

when

$$
f(p) \fallingdotseq h(x)
$$

$$
f(p)=p \int_{0}^{\infty} e^{-p x h} h(x) d x
$$

and

$$
\phi(p) \frac{K}{\bar{K}} h(x)
$$

when

$$
\phi(p)=\sqrt{ }(2 / \pi) p \int_{0}^{\infty}(p x)^{ \pm} K_{v}(p x) h(x) d x
$$

(2) is a generalisation of (1) as given by Meijer [2] and it reduces to (1) when $\nu= \pm \frac{1}{2}$ by virtue of the relation

$$
K_{ \pm \mathbf{l}}(z)=(\pi / 2 z)^{\mathbf{t}} e^{-z}
$$

In $(1), f(p)$ is called the image of $h(x)$ which is known as the original. The following abbreviations will be used.

$$
\begin{aligned}
\Gamma_{*}(a \pm b) & \equiv \Gamma(a+b) \Gamma(a-b), \\
\Gamma_{*}\left(a_{ \pm} b_{ \pm}\right) & \equiv \Gamma(a+b+c) \Gamma(a+b-c) \Gamma(a-b+c) \Gamma(a-b-c) .
\end{aligned}
$$

2. Theorem.

$$
\begin{array}{lc}
\text { If } & f(p) \fallingdotseq h(x) \\
\text { and } & \phi(p) \frac{K}{\bar{K}} x^{-t h(x),} \\
\text { then } & \int_{0}^{\infty} x^{-\nu-1}(x+z / x)^{-1} f(x+z / x) d x=\frac{1}{2} \sqrt{\pi} z^{-1 \nu-t} \phi(2 \sqrt{z}), \\
\text { or, } & \int_{0}^{\infty} \cosh \nu \theta \operatorname{sech} \theta f(p \cosh \theta) d \theta=(\pi / 2 p)^{ \pm} \phi(p) \\
\text { and } & \int_{0}^{\infty} \cosh \nu \theta \operatorname{sech} \theta h(x \operatorname{sech} \theta) d \theta=(\pi / 2)^{\ddagger} F(x), \ldots \ldots \ldots
\end{array}
$$

when the integrals are convergent and $F(x) \fallingdotseq p^{-\mathbf{t}} \phi(p)$.

Proof. We have

$$
f(p)=p \int_{0}^{\infty} e^{-p t} h(t) d t
$$

Therefore, $\quad \int_{0}^{\infty} x^{-v-1}(x+z / x)^{-1} f(x+z / x) d x=\int_{0}^{\infty} x^{-v-1}\left\{\int_{0}^{\infty} e^{-(x+z / x) t} h(t) d t\right\} d x$ 


$$
\begin{aligned}
& =\int_{0}^{\infty} h(t)\left\{\int_{0}^{\infty} x^{-y-1} e^{-(x+z / x) t} d x\right\} d t \\
& =2 z^{-i v} \int_{0}^{\infty} h(t) K_{v}(2 \sqrt{z} t) d t \\
& =\frac{1}{2} \sqrt{\pi} z^{-t v-t} \phi(2 \sqrt{z}), \quad R(z)>0,
\end{aligned}
$$

on changing the order of integration which we suppose to be permissible and using a wellknown integral $[4, \mathrm{p} .183]$.

(4) is obtained from (3) by the substitution $x=\sqrt{z} e^{\theta}$ and then replacing $2 \sqrt{z}$ by $p$, and (5) follows from (4) on finding the originals of both sides.

3. (i) From the integral $[1$, ex. 14, p. 345$]$

$$
\int_{0}^{\infty} e^{-p x} K_{n+\frac{1}{3}}(x) x^{m-1} d x=\sqrt{ }\left(\frac{1}{2} \pi\right) \Gamma(m+n+1) \Gamma(m-n)\left(p^{2}-1\right)^{-1 m} P_{n}^{m}(p)
$$

we find that

$$
\begin{aligned}
h(x)=x^{m-1} K_{n+1}(x) & \fallingdotseq \sqrt{ }\left(\frac{1}{2} \pi\right) \Gamma(m+n+1) \Gamma(m-n) p\left(p^{2}-1\right)^{-1 m} P_{n}^{-m}(p) \\
& =f(p), \quad R(m+n+1)>0, \quad R(m-n)>0,
\end{aligned}
$$

and from $[1$, ex. 87, p. 367$]$

$$
\begin{aligned}
& \int_{0}^{\infty} K_{v}(p x) K_{n}(x) x^{l-1} d x \\
= & \frac{\Gamma_{*}\left(\frac{1}{2} l \pm \frac{1}{2} \nu \pm \frac{1}{2} n\right)}{\Gamma(l)} 2^{l-3} p^{-l-n_{2}} F_{1}\left(\frac{1}{2} l+\frac{1}{2} \nu+\frac{1}{2} n, \frac{1}{2} l-\frac{1}{2} \nu+\frac{1}{2} n ; l ; 1-p^{-2}\right)
\end{aligned}
$$

we get

$$
\begin{aligned}
x^{-l} h(x)= & x^{m-1} K_{n+\frac{j}{3}}(x) \\
& \frac{K}{\bar{K}} \frac{\Gamma_{*}\left(\frac{1}{2} m \pm \frac{1}{2} \nu+\frac{1}{2} n+\frac{1}{2}\right) \Gamma_{*}\left(\frac{1}{2} m \pm \frac{1}{2} \nu-\frac{1}{2} n\right)}{\Gamma\left(\frac{1}{2}\right) \Gamma\left(m+\frac{1}{2}\right)} 2^{m-2} p^{t-m-n} \\
& \quad{ }_{2} F_{1}\left(\frac{1}{2} m+\frac{1}{2} \nu+\frac{1}{2} n+\frac{1}{2}, \frac{1}{2} m-\frac{1}{2} \nu+\frac{1}{2} n+\frac{1}{2} ; m+\frac{1}{2} ; 1-p^{-2}\right) \\
= & \phi(p), \quad R(m \pm \nu+n+1)>0, R(m \pm \nu-n)>0 .
\end{aligned}
$$

Applying (3) and (4) we obtain

$$
\begin{aligned}
& \int_{0}^{\infty} x^{-v-1}\left\{(x+z / x)^{2}-1\right\}^{-\frac{1}{2} m} P_{n}^{-m}(x+z / x) d x \\
& =\frac{\Gamma_{*}\left(\frac{1}{2} m \pm \frac{1}{2} \nu+\frac{1}{2} n+\frac{1}{2}\right) \Gamma_{*}\left(\frac{1}{2} m \pm \frac{1}{2} \nu-\frac{1}{2} n\right)}{\Gamma\left(\frac{1}{2}\right) \Gamma\left(m+\frac{1}{2}\right) \Gamma(m+n+1) \Gamma(m-n)} 2^{-n-2} z^{-1}(v+m+n+1) \\
& \quad \times_{2} F_{1}\left\{\frac{1}{2} m+\frac{1}{2} \nu+\frac{1}{2} n+\frac{1}{2}, \frac{1}{2} m-\frac{1}{2} \nu+\frac{1}{2} n+\frac{1}{2} ; m+1 ; 1-(4 z)^{-1}\right\}, \\
& R(m \pm v+n+1)>0, \quad R(m \pm \nu-n)>0, \quad R(z)>0, \quad\left|1-(4 z)^{-2}\right|<1 .
\end{aligned}
$$

$\int_{0}^{\infty} \cosh \nu \theta\left(p^{2} \cosh ^{2} \theta-1\right)^{-1 m} P_{n}^{-m}(p \cosh \theta) d \theta$

$$
\begin{aligned}
= & \frac{\Gamma_{*}\left(\frac{1}{2} m \pm \frac{1}{2} \nu+\frac{1}{2} n+\frac{1}{2}\right) \Gamma_{*}\left(\frac{1}{2} m \pm \frac{1}{2} \nu-\frac{1}{2} n\right)}{\Gamma\left(\frac{1}{2}\right) \Gamma\left(m+\frac{1}{2}\right) \Gamma(m+n+1) \Gamma(m-n)} p^{-m-n-1} 2^{m-2} \\
& \quad{ }_{2} F_{1}\left(\frac{1}{2} m+\frac{1}{2} \nu+\frac{1}{2} n+\frac{1}{2}, \frac{1}{2} m-\frac{1}{2} \nu+{ }_{2}^{1} n+\frac{1}{2} ; m+\frac{1}{2} ; 1-p^{-2}\right),
\end{aligned}
$$


$R(m \pm \nu+n+1)>0, \quad R(m \pm \nu-n)>0, \quad R(p)>0, \quad\left|1-p^{-2}\right|<1$.

If we take $\nu=\frac{1}{2}$ and use the relation [1, (24), p. 321]

we find that

$$
P_{n}^{-m}(z)=\frac{\left(z^{2}-1\right)^{\frac{1 m}{2}-m}}{z^{m+n+1} \Gamma(m+1)}{ }_{2} F_{1}\left(\frac{1}{2} m+\frac{1}{2} n+\frac{1}{2}, \frac{1}{2} m+\frac{1}{2} n+1 ; m+1 ; 1-z^{-2}\right),
$$

$\int_{0}^{\infty} \cosh \left(\frac{1}{2} \theta\right)\left(p^{2} \cosh ^{2} \theta-1\right)^{-1 m} P_{n}^{-m}(p \cosh \theta) d \theta$

$$
=\sqrt{\frac{\pi}{2 p}} \frac{\Gamma\left(m+n+\frac{1}{2}\right) \Gamma\left(m-n-\frac{1}{2}\right)}{\Gamma(m+n+1) \Gamma(m-n)}\left(p^{2}-1\right)^{t-i m} p_{n}^{t-m}(p),
$$

$R\left(m+n+\frac{1}{2}\right)>0, \quad R\left(m-n-\frac{1}{2}\right)>0, \quad R(p)>1$.

When $p \rightarrow 1$, (9) gives

$\int_{0}^{\infty} \cosh \nu \theta(\sinh \theta)^{-m} P_{n}-m(\cosh \theta) d \theta=\frac{\Gamma_{*}\left(\frac{1}{2} m \pm \frac{1}{2} \nu+\frac{1}{2} n+\frac{1}{2}\right) \Gamma_{*}\left(\frac{1}{2} m \pm \frac{1}{2} \nu-\frac{1}{2} n\right)}{\Gamma\left(\frac{1}{2}\right) \Gamma\left(m+\frac{1}{2}\right) \Gamma(m+n+1) \Gamma(m-n)} 2^{m-2}$,

$R(m \pm \nu+n+1)>0, \quad R(m \pm \nu-n)>0$.

Since

${ }_{2} F_{1}(a, b ; c ; z)=\frac{\Gamma(c) \Gamma(c-a-b)}{\Gamma(c-a) \Gamma(c-b)}{ }_{2} F_{1}(a, b ; a+b-c+1 ; 1-z)$

we have

$$
+\frac{\Gamma(c) \Gamma(a+b-c)}{\Gamma(a) \Gamma(b)}(1-z)^{c-a-b}{ }_{2} F_{1}(c-a, c-b ; c-a-b+1 ; 1-z),
$$

$$
\begin{aligned}
& p^{-+} \phi(p)=\frac{\Gamma_{*}\left(\frac{1}{2} m \pm \frac{1}{2} \nu+\frac{1}{2} n+\frac{1}{2}\right) \Gamma_{*}\left(\frac{1}{2} m \pm \frac{1}{2} \nu-\frac{1}{2} n\right)}{\Gamma\left(\frac{1}{2}\right) \Gamma\left(m+\frac{1}{2}\right)} 2^{m-2} p^{-m-n} \\
& \times_{2} F_{1}\left(\frac{1}{2} m+\frac{1}{2} \nu+\frac{1}{2} n+\frac{1}{2}, \frac{1}{2} m-\frac{1}{2} \nu+\frac{1}{2} n+\frac{1}{2} ; m+\frac{1}{2} ; 1-p^{-2}\right) \\
& =\frac{2^{m-2}}{\Gamma\left(\frac{1}{2}\right)}\left[\Gamma_{*}\left(\frac{1}{2} m \pm \frac{1}{2} \nu+\frac{1}{2} n+\frac{1}{2}\right) \Gamma\left(-n-\frac{1}{2}\right) p^{-m-n}\right. \\
& \times{ }_{2} F_{1}\left(\frac{1}{2} m+\frac{1}{2} \nu+\frac{1}{2} n+\frac{1}{2}, \frac{1}{2} m-\frac{1}{2} \nu+\frac{1}{2} n+\frac{1}{2} ; n+\frac{3}{2} ; p^{-2}\right) \\
& +\Gamma_{*}\left(\frac{1}{2} m \pm \frac{1}{2} \nu-\frac{1}{2} n\right) \Gamma\left(n+\frac{1}{2}\right) p^{n-m+1} \\
& \left.\times_{2} F_{1}\left(\frac{1}{2} m+\frac{1}{2} v-\frac{1}{2} n, \frac{1}{2} m-\frac{1}{2} v-\frac{1}{2} n ; \frac{1}{2}-n ; p^{-2}\right)\right] \\
& \fallingdotseq \frac{2^{m-2}}{\Gamma\left(\frac{1}{2}\right)}\left[\frac{\Gamma_{*}\left(\frac{1}{2} m \pm \frac{1}{2} \nu+\frac{1}{2} n+\frac{1}{2}\right) \Gamma\left(-n-\frac{1}{2}\right)}{\Gamma(m+n+1)} x^{m+n}\right. \\
& \times{ }_{2} F_{3}\left(\frac{1}{2} m+\frac{1}{2} \nu+\frac{1}{2} n+\frac{1}{2}, \frac{1}{2} m-\frac{1}{2} \nu+{ }_{2}^{1} n+\frac{1}{2} ; \quad \frac{1}{2} m+\frac{1}{2} n+\frac{1}{2}, \frac{1}{2} m+\frac{1}{2} n+1, n+\frac{3}{2} ; 4 x^{2}\right) \\
& +\frac{\Gamma_{*}\left(\frac{1}{2} m \pm \frac{1}{2} \nu-\frac{1}{2} n\right) \Gamma\left(n+\frac{1}{2}\right)}{\Gamma(m-n)} x^{m-n-1} \\
& \left.\times_{2} F_{3}\left(\frac{1}{2} m+\frac{1}{2} \nu-\frac{1}{2} n, \frac{1}{2} m-\frac{1}{2} \nu-\frac{1}{2} n ; \frac{1}{2} m-\frac{1}{2} n, \frac{1}{2} m-\frac{1}{2} n+\frac{1}{2}, \frac{1}{2}-n ; \frac{1}{6} x^{2}\right)\right] \\
& =F(x) \text {. }
\end{aligned}
$$


Hence applying (5) we have

$\int_{0}^{\infty} \cosh \nu \theta(\operatorname{sech} \theta)^{m+1} K_{n+1}(x \operatorname{sech} \theta) d \theta$

$$
\begin{gathered}
=\frac{\Gamma_{*}\left(\frac{1}{2} m \pm \frac{1}{2} \nu+\frac{1}{2} n+\frac{1}{2}\right) \Gamma\left(-n-\frac{1}{2}\right)}{\Gamma(m+n+1)} x^{n+1} 2^{m-\frac{5}{2}} \\
\quad \times{ }_{2} F_{3}\left(\frac{1}{2} m+\frac{1}{2} \nu+\frac{1}{2} n+\frac{1}{2}, \frac{1}{2} m-\frac{1}{2} \nu+\frac{1}{2} n+\frac{1}{2} ; \quad \frac{1}{2} m+\frac{1}{2} n+\frac{1}{2}, \frac{1}{2} m+\frac{1}{2} n+1, n+\frac{3}{2} ; \quad \frac{1}{2} x^{2}\right) \\
+\frac{\Gamma_{*}\left(\frac{1}{2} m \pm \frac{1}{2} \nu-\frac{1}{2} n\right) \Gamma\left(n+\frac{1}{2}\right)}{\Gamma(m-n)} x^{n+1} 2^{m-\frac{x}{2}} \\
\quad \times{ }_{2} F_{3}\left(\frac{1}{2} m+\frac{1}{2} \nu-\frac{1}{2} n, \frac{1}{2} m-\frac{1}{2} \nu-\frac{1}{2} n ; \quad \frac{1}{2} m-\frac{1}{2} n, \frac{1}{2} m-\frac{1}{2} n+\frac{1}{2}, \frac{1}{2}-n ; 1 x^{2}\right), \ldots \ldots(13) \\
R(m \pm \nu+n+1)>0, \quad R(m \pm \nu-n)>0, \quad R(x)>0 .
\end{gathered}
$$

(ii) From the formula $[1,(89)$, p. 342]

we have

$$
\int_{0}^{\infty} e^{-p x} I_{n+\xi}(x) x^{m-1} d x=\sqrt{ }(2 / \pi)\left(p^{2}-1\right)^{-1 m} Q_{n}^{m}(p),
$$

$$
\begin{aligned}
h(x) & =x^{m-1} I_{n+\xi}(x) \\
& \fallingdotseq \sqrt{ }(2 / \pi) p\left(p^{2}-1\right)^{-\mathrm{i} m} Q_{n}^{m}(p) \ldots \ldots \ldots \ldots . . \\
& =f(p), \quad R(m+n+1)>0, \quad R(p)>1,
\end{aligned}
$$

and from $[1$, ex. 88, p. 367]

$$
\int_{0}^{\infty} K_{v}(p x) I_{n}(x) x^{l-1} d x=\frac{\Gamma_{*}\left(\frac{1}{2} l \pm \frac{1}{2} \nu+\frac{1}{2} n\right)}{\Gamma(n+1)} 2^{l-2} p^{-l-n}{ }_{2} F_{1}\left(\frac{1}{2} l+\frac{1}{2} \nu+\frac{1}{2} n, \frac{1}{2} l-\frac{1}{2} v+\frac{1}{2} n ; \quad n+1 ; p^{-2}\right),
$$

we get

$$
\begin{aligned}
x^{-1} h(x) & =x^{m-1} I_{n+\frac{1}{1}}(x) \\
\frac{K}{\bar{B}} \frac{\Gamma_{*}\left(\frac{1}{2} m \pm \frac{1}{2} \nu+\frac{1}{2} n+\frac{1}{2}\right)}{\Gamma\left(\frac{1}{2}\right) \Gamma\left(n+\frac{3}{2}\right)} 2^{m-1} p^{\frac{1}{2}-m-n} & \quad{ }_{2} F_{1}\left(\frac{1}{2} m+\frac{1}{2} \nu+\frac{1}{2} n+\frac{1}{2}, \frac{1}{2} m-\frac{1}{2} \nu+\frac{1}{2} n+\frac{1}{2} ; n+\frac{3}{2} ; p^{-2}\right) \\
& =\phi(p), \quad R(m \pm \nu+n+1)>0, \quad R(p)>1 .
\end{aligned}
$$

Hence (3) and (4) give

$$
\begin{aligned}
& \int_{0}^{\infty} x^{-v-1}\left\{(x+z / x)^{2}-1\right\}^{-\frac{1}{m}} Q_{n}^{m}(x+z / x) d x \\
& =\frac{\Gamma\left(\frac{1}{2}\right) \Gamma_{*}\left(\frac{1}{2} m \pm \frac{1}{2} \nu+\frac{1}{2} n+\frac{1}{2}\right)}{\Gamma\left(n+\frac{3}{2}\right)} 2^{-n-2} z^{-1(v+m+n+1)} \\
& \quad{ }_{2} F_{1}\left(\frac{1}{2} m+\frac{1}{2} \nu+\frac{1}{2} n+\frac{1}{2}, \frac{1}{2} m-\frac{1}{2} \nu+\frac{1}{2} n+\frac{1}{2} ; n+\frac{3}{2} ; 1 / 4 z\right),
\end{aligned}
$$

$R(m \pm \nu+n+1)>0, \quad R(z)>\frac{1}{4}$.

$\int_{0}^{\infty} \cosh \nu \theta\left\{(p \cosh \theta)^{2}-1\right\}^{-\mathrm{d} m} Q_{n}^{m}(p \cosh \theta) d \theta$

$$
\begin{aligned}
=\frac{\Gamma\left(\frac{1}{2}\right) \Gamma_{*}\left(\frac{1}{2} m \pm \frac{1}{2} \nu+\frac{1}{2} n+\frac{1}{2}\right)}{\Gamma\left(n+\frac{3}{2}\right)} 2^{m-2} p^{-m-n-1} & \\
& \quad{ }_{2} F_{1}\left(\frac{1}{2} m+\frac{1}{2} \nu+\frac{1}{2} n+\frac{1}{2}, \frac{1}{2} m-\frac{1}{2} v+\frac{1}{2} n+\frac{1}{2} ; n+\frac{3}{2} ; p^{-2}\right)
\end{aligned}
$$

$R(m \pm v+n+1)>0, \quad R(p)>1$. 
When $p \rightarrow 1$, we have

$\int_{0}^{\infty} \cosh \nu \theta(\sinh \theta)^{-m} Q_{n}^{m}(\cosh \theta) d \theta=\frac{\Gamma_{*}\left(\frac{1}{2} m \pm \frac{1}{2} \nu+\frac{1}{2} n+\frac{1}{2}\right) \Gamma\left(\frac{1}{2}-m\right) \Gamma\left(\frac{1}{2}\right)}{\Gamma_{*}\left(\frac{1}{2} n \pm \frac{1}{2} \nu-\frac{1}{2} m+1\right)} 2^{m-2}$,

$R(m \pm \nu+n+1)>0, \quad R(m)<\frac{1}{2}$.

If we take $\nu=\frac{1}{2}$ in (17) and use the relation $[1,(9)$, p. 316]

$Q_{n}^{m}(z)=\frac{\Gamma\left(\frac{1}{2}\right) \Gamma(n+m+1)\left(z^{2}-1\right)^{\frac{1}{m}}}{2^{n+1} \Gamma\left(n+\frac{3}{2}\right) z^{n+m+1}}{ }_{2} F_{1}\left(\frac{1}{2} n+\frac{1}{2} m+\frac{1}{2}, \frac{1}{2} n+\frac{1}{2} m+1 ; n+\frac{3}{2} ; z^{-2}\right)$,

we get

$\int_{0}^{\infty} \cosh \left(\frac{1}{2} \theta\right)\left\{(p \cosh \theta)^{2}-1\right\}^{-\frac{1}{2} m} Q_{n}^{m}(p \cosh \theta) d \theta=\sqrt{ }(\pi / 2)\left(p^{2}-1\right)^{t-\frac{1}{2} m} Q_{n}^{m-1}(p)$, $R\left(m+n+\frac{1}{2}\right)>0, \quad R(p)>1$.

Also,

$$
\begin{aligned}
p^{-i} \phi(p)= & \frac{\Gamma_{*}\left(\frac{1}{2} m \pm \frac{1}{2} \nu+\frac{1}{2} n+\frac{1}{2}\right)}{\Gamma\left(\frac{1}{2}\right) \Gamma\left(n+\frac{3}{2}\right)} 2^{m-1} p^{-m-n} \\
& \quad \times{ }_{2} F_{1}\left(\frac{1}{2} m+\frac{1}{2} \nu+\frac{1}{2} n+\frac{1}{2}, \frac{1}{2} m-\frac{1}{2} \nu+\frac{1}{2} n+\frac{1}{2} ; n+\frac{3}{2} ; p^{-2}\right) \\
\fallingdotseq & \frac{\Gamma_{*}\left(\frac{1}{2} m \pm \frac{1}{2} \nu+\frac{1}{2} n+\frac{1}{2}\right)}{\Gamma\left(\frac{1}{2}\right) \Gamma\left(n+\frac{3}{2}\right) \Gamma(m+n+1)} 2^{m-1} x^{m+n} \\
\quad & \quad{ }_{2} F_{3}\left(\frac{1}{2} m+\frac{1}{2} \nu+\frac{1}{2} n+\frac{1}{2}, \frac{1}{2} m-\frac{1}{2} \nu+\frac{1}{2} n+\frac{1}{2} ; 2 \frac{1}{2} m+\frac{1}{2} n+\frac{1}{2}, \frac{1}{2} m+\frac{1}{2} n+1, n+\frac{3}{2} ; \frac{1}{2} x^{2}\right) \\
= & F(x) .
\end{aligned}
$$

Hence (5) gives

$\int_{0}^{\infty} \cosh \nu \theta(\operatorname{sech} \theta)^{m+1} I_{n+\frac{1}{\xi}}(x \operatorname{sech} \theta) d \theta$

$$
\begin{aligned}
= & \frac{\Gamma_{*}\left(\frac{1}{2} m \pm \frac{1}{2} \nu+\frac{1}{2} n+\frac{1}{2}\right)}{\Gamma\left(n+\frac{3}{2}\right) \Gamma(m+n+1)} 2^{m-\frac{3}{3}} x^{n+\frac{1}{2}} \\
& \times{ }_{2} F_{3}\left(\frac{1}{2} m+\frac{1}{2} \nu+\frac{1}{2} n+\frac{1}{2}, \frac{1}{2} m-\frac{1}{2} \nu+\frac{1}{2} n+\frac{1}{2} ; \frac{1}{2} m+\frac{1}{2} n+\frac{1}{2}, \frac{1}{2} m+\frac{1}{2} n+1, n+\frac{3}{2} ; \quad \frac{1}{2} x^{2}\right),
\end{aligned}
$$
$R(m \pm v+n+1)>0, R(x)>0$.

(iii) Writing the integral [1, ex. 8, p. 344]

$$
\int_{0}^{\infty} e^{-\lambda x} K_{m}\left\{\lambda \sqrt{x^{2}-1}\right\} \lambda^{n} d \lambda=\Gamma(n+m+1) Q_{n}^{-m}(x)
$$

in the form

$$
\int_{0}^{\infty} e^{-p t} t^{n} K_{m}(t) d t=\Gamma(n-m+1)\left(p^{2}-1\right)^{-t n-t} Q_{n}^{m}\left\{p / \sqrt{ }\left(p^{2}-1\right)\right\}
$$

we find that

$$
\begin{aligned}
h(x) & =x^{n} K_{m}(x) \\
& \fallingdotseq \Gamma(n-m+1) p\left(p^{2}-1\right)^{-1 n-1} Q_{n}^{m}\left\{p / \sqrt{ }\left(p^{2}-1\right)\right\} \\
& =f(p), \quad R(n \pm m+1)>0, \quad R(p)>1,
\end{aligned}
$$


and from (7) we have

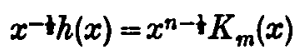

$$
\begin{aligned}
& \frac{K}{\bar{K}} \frac{\Gamma_{*}\left(\frac{1}{2} n \pm \frac{1}{2} \nu \pm \frac{1}{2} m+\frac{1}{2}\right)}{\Gamma(n+1) \Gamma\left(\frac{1}{2}\right)} 2^{n-\frac{s}{2}} p^{-n-m} \\
& \times{ }_{2} F_{1}\left(\frac{1}{2} n+\frac{1}{2} v+\frac{1}{2} m+\frac{1}{2}, \frac{1}{2} n-\frac{1}{2} v+\frac{1}{2} n+\frac{1}{2} ; n+1 ; 1-p^{-2}\right)
\end{aligned}
$$

$=\phi(p), \quad R(n \pm \nu \pm m+1)>0$.

Applying (3) and (4) we get

$$
\begin{aligned}
& \int_{0}^{\infty} x^{-\nu-1}\left\{(x+z / x)^{2}-1\right\}^{-1 n-1} Q_{n}^{m}\left[(x+z / x) / \sqrt{ }\left\{(x+z / x)^{2}-1\right\}\right] d x \\
& =\frac{\Gamma_{*}\left(\frac{1}{2} n \pm \frac{1}{2} \nu \pm \frac{1}{2} m+\frac{1}{2}\right)}{\Gamma(n+1) \Gamma(n-m+1)} z^{-\frac{1}{(1)+m+n+1)} 2^{-m-2}} \\
& \quad{ }_{2} F_{1}\left\{\frac{1}{2} n+\frac{1}{2} \nu+\frac{1}{2} m+\frac{1}{2}, \frac{1}{2} n-\frac{1}{2} \nu+\frac{1}{2} m+\frac{1}{2} ; n+1 ; 1-(4 z)^{-1}\right\}
\end{aligned}
$$

$R(n \pm \nu \pm m+1)>0, \quad R(z)>\frac{1}{8}$, and

$\int_{0}^{\infty} \cosh \nu \theta\left(p^{2} \cosh ^{2} \theta-1\right)^{-\frac{1}{n} n-1} Q_{n}^{m}\left\{p \cosh \theta / \sqrt{ }\left(p^{2} \cosh ^{2} \theta-1\right\} d \theta\right.$

$$
\begin{aligned}
=\frac{\Gamma_{*}\left(\frac{1}{2} n \pm \frac{1}{2} \nu \pm \frac{1}{2} m+\frac{1}{2}\right)}{\Gamma(n-m+1) \Gamma(n+1)} & 2^{n-2} p^{-n-m-1} \\
& \times_{2} F_{1}\left(\frac{1}{2} n+\frac{1}{2} \nu+\frac{1}{2} m+\frac{1}{2}, \frac{1}{2} n-\frac{1}{2} \nu+\frac{1}{2} m+\frac{1}{2} ; n+1 ; 1-p^{-2}\right),
\end{aligned}
$$

$R(n \pm \nu \pm m+1)>0, \quad\left|p^{2}-1\right|<\left|p^{2}\right|$.

If we take $\nu=\frac{1}{2}$ and use (19) we have

$\int_{0}^{\infty} \cosh \left(\frac{1}{2} \theta\right)\left(p^{2} \cosh ^{2} \theta-1\right)^{-\frac{1}{n} n-t} Q_{n}^{m}\left\{p \cosh \theta / \sqrt{ }\left(p^{2} \cosh ^{2} \theta-1\right)\right\} d \theta$

$$
=\sqrt{\frac{\pi}{2 p}} \frac{\Gamma\left(n-m+\frac{1}{2}\right)}{\Gamma(n-m+1)}\left(p^{2}-1\right)^{-\frac{n}{2}-\frac{1}{4}} Q_{n-4}^{m}\left\{p / \sqrt{ }\left(p^{2}-1\right)\right\},
$$

$R\left(n \pm m+\frac{1}{2}\right)>0, \quad\left|p^{2}-1\right|<\left|p^{2}\right|$.

When $p \rightarrow 1$, (25) yields

$$
\begin{aligned}
& \int_{0}^{\infty} \cosh \nu \theta(\sinh \theta)^{-n-1} Q_{n}^{m}(\operatorname{coth} \theta) d \theta=\frac{\Gamma_{*}\left(\frac{1}{2} n \pm \frac{1}{2} \nu \pm \frac{1}{2} m+\frac{1}{2}\right)}{\Gamma(n-m+1) \Gamma(n+1)} 2^{n-2}, \\
& R(n \pm \nu \pm m+1)>0 .
\end{aligned}
$$
formula

Note.-Results (24) to (27) may also be derived from (8) to (12) by applying Whipple's

$$
P_{n}^{-m}(p)=\sqrt{\frac{2}{\pi}} \frac{\left(p^{2}-1\right)^{-t}}{\Gamma(m+n+1)} Q_{m-1}^{n+1}\left\{p / \sqrt{ }\left(p^{2}-1\right)\right\} .
$$

(iv) From the integral $[3$, p. 119]

$\int_{0}^{\infty} K_{v}(x) x^{\gamma-1} E\left(l ; \alpha_{r}: m ; \rho_{s}: z / x^{2 n}\right) d x=(2 \pi)^{1-n} 2^{\gamma-2} n^{\gamma-1} E\left\{l+2 n ; \alpha_{r}: m ; \rho_{s}: z /(2 n)^{2 n}\right\}$ 
we have

$$
\begin{aligned}
& \begin{aligned}
& x^{-t h}(x)=x^{\gamma-\frac{s}{2}} E\left(l ; \alpha_{r}: m ; \rho_{s}: 1 / x^{2 n}\right) \\
& \frac{K}{\bar{K}} \sqrt{ }(2 / \pi)(2 \pi)^{1-n} 2^{\gamma-2} n^{\gamma-1} p^{\frac{3}{2}-\gamma} E\left\{l+2 n ; \alpha_{r}: m ; \rho_{s}:(p / 2 n)^{2 n}\right\} \\
&= \phi(p), \\
& R(\gamma \pm \nu)>0, \quad \alpha_{l+k+1}=(\gamma+\nu+2 k) / 2 n, \\
& \quad \alpha_{l+n+k+1}=(\gamma-\nu+2 k) / 2 n, \quad k=0,1, \ldots,(n-1) .
\end{aligned}
\end{aligned}
$$

Taking $\nu=\frac{1}{2}$ in the above and replacing $\gamma$ by $\gamma+\frac{1}{2}$, we have

$$
\begin{aligned}
h(x) & =x^{\gamma-1} E\left(l ; \alpha_{r}: m ; \rho_{s}: 1 / x^{2 n}\right) \\
& \fallingdotseq \sqrt{ }(2 / \pi)(2 \pi)^{1-n} 2^{\gamma-\frac{3}{8}} n^{\gamma-i} p^{1-\gamma} E\left\{l+2 n ; \alpha_{r}^{*}: m ; \rho_{s}:(p / 2 n)^{2 n}\right\} \\
& =f(p),
\end{aligned}
$$

$R(\gamma)>0, \alpha_{q}^{*}=\alpha_{0}, q=1,2, \ldots, l ; \alpha_{l+k+1}^{*}=(\gamma+1+2 k) / 2 n, \alpha_{l+n+k+1}^{*}=(\gamma+2 k) / 2 n, k=0,1, \ldots$, $(n-1)$.

Applying (3) and (4) we get

$$
\begin{aligned}
\int_{0}^{\infty} x^{-\nu-1}(x+z / x)^{-\gamma} & E\left[l+2 n ; \alpha_{r}^{*}: m ; \rho_{s}:\{(x+z / x) / 2 n\}^{2 n}\right] d x \\
& =\sqrt{ }(\pi / n) 2^{-\gamma_{z}} z^{-\downarrow \nu-\downarrow \gamma} E\left\{l+2 n ; \alpha_{r}: m ; \rho_{s}:\left(z / n^{2}\right)^{n}\right\}
\end{aligned}
$$

$R(\gamma \pm \nu)>0, \quad R(z)>0$.

$$
\begin{aligned}
\int_{0}^{\infty} \cosh \nu \theta(\cosh \theta)^{-\gamma} & \left\{l l+2 n ; \alpha_{r}^{*}: m ; \rho_{s}:(p \cosh \theta / 2 n)^{2 n}\right\} d \theta \\
& =\frac{1}{2} \sqrt{ }(\pi / n) E\left\{l+2 n ; \alpha_{r}: m ; \rho_{s}:(p / 2 n)^{2 n}\right\},
\end{aligned}
$$

$R(\gamma \pm v)>0, \quad R(p)>0$.

Results (30) and (31) may be put in a more compact form thus :

$$
\begin{aligned}
\int_{0}^{\infty} x^{-\nu-1}(x+z / x)^{-\gamma} E\left[l ; \alpha_{r}: m ; \rho_{s}:\{(x+z / x) / 2 n\}^{2 n}\right] d x \\
=\sqrt{ }(\pi / n) 2-\gamma_{z}-\mathbf{l} v-\downarrow \gamma E\left\{l+2 n ; \alpha_{r}: m+2 n ; \rho_{s}:\left(z / n^{2}\right)^{n}\right\},
\end{aligned}
$$

$R(\gamma \pm v)>0, R(z)>0$.

$$
\int_{0}^{\infty} \cosh \nu \theta(\cosh \theta)^{-\gamma} E\left\{l ; \alpha_{r}: m ; \rho_{s}: \lambda(\cosh \theta)^{2 n}\right\} d \theta
$$

$$
=\frac{1}{2} \sqrt{ }(\pi / n) E\left(l+2 n ; \alpha_{r}: m+2 n ; \rho_{s}: \lambda\right) \text {, }
$$

$R(\gamma \pm \nu)>0, R(\lambda)>0, \alpha_{l+k+1}=(\gamma+\nu+2 k) / 2 n, \alpha_{l+n+k+1}=(\gamma-\nu+2 k) / 2 n, \rho_{m+k+1}=(\gamma+1+2 k) / 2 n$ $\rho_{n+n+k+1}=(\gamma+2 k) / 2 n, k=0,1, \ldots,(n-1)$.

\section{REFERENCES}

(1) MacRobert, T. M., Spherical Harmonics, Methuen (1947).

(2) Meijer, C. S., "Uber eine Erweiterung der Laplace-Transformation ", Proc. Kon. Neder. Akad. van Wetenschappen, (5), 43 (1940).

(3) Ragab, F. M., Proc. Glasg. Math. Assn., Pt. III, 1 (1953).

(4) Watson, G. N., Theory of Bessel Functions, (1944).

Maharana Bhopal College

\section{UDAIPUR}

